\title{
Impact of Advertisements during Channel Zapping on Quality of Experience
}

\author{
B.E. Godana ${ }^{1}$, R.E. Kooij ${ }^{1,2}$, O.K. Ahmed ${ }^{2}$ \\ ${ }^{1}$ University of Technology Delft, Fac. of Electrical Engineering, Mathematics and Computer \\ Science \\ ${ }^{2}$ TNO Information and Communication Technology, Delft, the Netherlands, \\ \{robert.kooij,kamal.ahmed\}@tno.nl
}

\begin{abstract}
Nowadays various digital television services are available. However, the user of these services experiences longer delays than the traditional analog $T V$ while switching from channel to channel. The digital TV operator usually displays a black screen with the channel number during zapping. However, it could be interesting for the TV viewer, if the operator displays a screen with information instead of just a black screen. This information may be an advertisement, information about the target channel, personalized content of the user etc. In this paper, we describe a subjective experiment where the Quality of Experience (QoE) of channel zapping was quantified, while displaying a random set of advertisement pictures during zapping. It is found that, for longer zapping times, advertisements give better QoE than the black screen. However, when zapping times are small,users prefer a black screen over a glance of an advertisement picture.
\end{abstract}

\section{Introduction}

Telecom Service Providers around the world are in a race to deploy new revenue generating services in order to offset the accelerating decline in voice revenues. For instance, US based providers faced a decline of $34 \%$ in voice-related revenues between 2000 and 2008 [8]. One of the new services Service Providers came up with is "triple play", which is the commercial bundling of voice, video and data on a common IP based network infrastructure. This IP based network infrastructure allows providing enhanced applications and services such as IPTV, VoIP, video telephony and Video on demand (VoD). However, as providers deploy new services, they also have to provide optimal Quality of Experience (QoE).
QoE takes into account how well a service meets customers goals and expectations rather than focusing only on the network performance. In this highly competitive market Service Providers which are offering high quality IPTV services should address the QoE requirements of IPTV.

One of the key elements of QoE of IPTV is how quickly users can change between TV channels, which is called channel zapping. Minimum quality requirements for a lot of aspects related to IPTV have been specified by both the ITU (see [5]) and the DSL Forum [3]. However in the ITU document there are no recommendations at all related to zapping times, while in the DSL forum document it is recommended to limit zapping time to an arbitrary maximum of 2 seconds. Additionally it is noticed in the document that providers should strive for zapping times in the order of 1 second.

Because these quality requirements are rather vague Kooij et al. [7] conducted a number of subjective tests in order to get insight in the relation between QoE and zapping time. For the tests described in [7], during channel zapping a black screen was visible which contained the number of the target channel. The QoE was expressed as a so-called Mean Opinion Score (MOS). The test subjects (21 in total) could select one of the following five opinion scores, motivated by the ITU-T ACR scale, see [6]: 5: Excellent zapping quality, 4: Good zapping quality, 3: Fair zapping quality, 2: Poor zapping quality, 1: Bad zapping quality.

The main result of [7] is an explicit relation between the user perceived QoE and the zapping time. From this relation it was deduced that in order to guarantee a MOS of at least 3.5, which is considered the lower bound for acceptable quality of service, see [6], we need to ascertain that Zapping Time $<430 \mathrm{~ms}$. 
This requirement on the zapping time is currently not met in any implementation of IPTV, see for instance [4]. To increase the QoE of channel zapping, two approaches are possible. In the first approach the actual zapping time is reduced. An example of this method is given by Degrande et al. [2]. They suggest to retain the most recent video part in a circular buffer and display this video until the incoming channel is ready.

In the second approach the QoE is (possibly) increased by showing information while the user waits for the target channel to appear. The displayed information could be about the target channel, personalized content or advertisements, see also [1].

The aim of this paper is to assess the QoE of channel zapping when, during zapping, advertisements are displayed, instead of the usual black screen.

The rest of this paper is organized as follows. In section 2, the possible effect of advertisements on IPTV perceived quality is analyzed and various factors that contribute to the results are listed. In Section 3 the experiment performed to quantify the user perception is described. In section 4, the results obtained from the subjective tests are presented. Finally, conclusions are given in Section 5.

\section{Quality of Experience and Advertisements}

\subsection{Quality of Experience of IPTV}

Quality of Experience is the quality as judged by the user. QoE for IPTV is a subjective measure of the IPTV service that is evaluated by test subjects and depends on two types of factors. The first type of factors is due to the actual Quality of Service (QoS) or network quality being provided. The other type of factors result in a change of the user perception even though the QoS being provided remains the same.

Some of the factors that affect the QoE resulting from the actual QoS of the network are,

a) The zapping time;

b) The visual quality: this factor depends on the quality of encoding and decoding and on the packet loss in the network;

c) Synchronization between video and audio.
The other factors which result in variation in the user perception, even though the QoS remains the same, are:

a) The user device: the equipment the user is using to watch the channel is also important, for instance, the screen resolution of the TV;

b) The educational level, age and the TV watching experience of the customers;

c) The mood and concentration of the customer;

d) Viewing conditions, such as room illumination, display type (brightness, contrast), viewing distance etc.;

e) The IPTV service cost.

Measuring the QoE is very important for the service provider. Once the quality perceived by the user is measured, the vendor can determine the minimum requirements on the IPTV service quality (such as the maximum tolerable zapping time). Moreover, the vendors can provide additional services or use techniques to boost the user perception with the same QoS level being provided. For example, using advertisements during channel zapping may increase the QoE.

\subsection{Effect of Advertisements on QoE}

Using advertisements during the IPTV zapping times is an approach that tries to increase the QoE while the service quality or zapping time remains unchanged. However, not all users may have the same perception for advertisements. For instance, there could be people who dislike advertisements. However, using advertisements during channel zapping could interest a large number of customers. There are two major consequences that are expected to boost the QoE in the case of the actual implementation of this approach.

a) Users will watch the advertisements during the channel zapping, so they will not be bored with the longer zapping times. Hence, the perception of the user for the channels with advertisements could increase with respect to the black screens. This is actually what we have measured in the conducted subjective experiment.

b) The second consequence is that the providers will earn money from these advertisements. So, they can lower the price of the service. Obviously, a lower price is one of the factors that can boost the QoE. 
It should be noted that the effect of advertisements on QoE is not just straightforward if it would be implemented. Rather, it depends on various factors which could affect the QoE positively or negatively.

a) The type of advertisement: A particular user could like some sort of advertisements and dislike other advertisements.

b) The content of the advertisement picture with respect to the length of the zapping time: For example, a glance of an advertisement that stays for a very short duration or a picture advertisement that stays static for a zapping time of $5 \mathrm{sec}$ could be annoying for the user. Advertisements containing much text or video advertisements may be of little importance if zapping time is short.

c) The advertisement between the channels may need to be made random for better user perception; moreover, the advertisement set should be changed after some time.

Some of the factors above could positively affect the user perception. However, the implementation complexity also increases if all these issues are to be properly addressed. The best approach to use these advertisements is to select an advertisement randomly from a set of advertisements pre-rendered and stored in the Set-top Box (STB) when the user zaps to a different channel. Using pre-rendered advertisements is important because the zap screen can then be displayed immediately in this case.

\section{The experiment}

\subsection{Design of the experiment}

For the IPTV channel zapping experiment, a HTML page containing five animated gifs in different layers is implemented in JavaScript. These five animated gifs correspond to 5 different TV channel contents: an orchestra scene, two film trailers, a cartoon scene, and a sports scene. These animated gifs do not contain audio. Audio can be added but the synchronization problem will be another cause for quality degradation. So, to assess the quality experienced for zapping times independently, it is better to make the experiments with no sound. The animated gifs are displayed in a screen of size of $720 \times 576$ pixels in the HTML page. The page is designed in layers such that when the user zaps to a particular channel all animated gif layers become invisible except the layer containing the required animated gif.

For this experiment, seven zapping times between 0 and 5 second are implemented in arrays in the javascript code. These zapping times are $0,0.1,0.2$, $0.2,0.5,0.5,1,2,2$ and $5 \mathrm{sec}$. Some of the zapping times are repeated to see the consistency of the users. Moreover, a random ordering of these zapping times is implemented for each of the 12 test subjects. When the user zaps to a new channel, the page sleeps for a time corresponding to the implemented zapping time before the requested channel is displayed. During this time a random advertisement picture is selected from a set of advertisement pictures and it is displayed. This chain of events is depicted in Figure 1. For all advertisements we have used logo-like pictures.

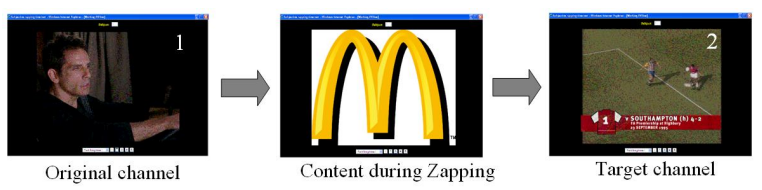

Figure 1: Showing an advertisement during zapping

When the user zaps to the next channel the same step is repeated, but an advertisement different from the advertisement shown during the previous zapping epoch is selected in random manner.

\subsection{The actual experiment}

For the Experiment 12 test subjects were selected. The test subjects consisted of 12 people at TNO ICT in Delft, the Netherlands. The test subjects varied in age, gender and experience.

To view the channels a laptop (Pentium 4, 2GB RAM, windows vista, $1500 \times 750$ pixels screen resolution) is used as a TV set. The experiment that we have conducted is of 'lean backward zapping' type. That means the user will sit back in a chair and use the remote control to zap between the channels. A Sony Ericsson Bluetooth enabled mobile phone is used as a remote control device. The experiment contains two parts, the training and the actual experiment.

In the training session, we show the test subjects three zapping times: instantaneous, intermediate and slow to give them an example of how the zapping times in the actual experiment are to be assessed. 
During this session, the test subject will get used to the ITU MOS scale.

During the actual experiment the test subjects were asked to experience the zapping times by zapping between the channels using the remote control (mobile phone) then to evaluate the experienced quality. They evaluate their perception for the ten zapping times, first using black screen and then using advertisements during zapping. The users are also given the chance to give open suggestions.

\section{Results}

\subsection{MOS results}

The results obtained for each zapping time are analysed and averaged over the twelve test subjects to obtain the MOS for each zapping time. This is done for both the case where a black screen is shown during zapping and the case where an advertisement is shown. From now on we will refer two these two cases as 'black screen' and 'advertisement'. The obtained results are shown in Table 1 and Table 2, respectively.

Table 1: MOS for 'black screen'

\begin{tabular}{|c|c|c|}
\hline Zapping Time(sec) & MOS & Std. Dev. \\
\hline 0 & 4.75 & 0.62 \\
\hline 0.1 & 4.83 & 0.39 \\
\hline 0.2 & 4.42 & 0.67 \\
\hline 0.2 & 4.25 & 0.62 \\
\hline 0.5 & 3.33 & 0.89 \\
\hline 0.5 & 3.50 & 0.67 \\
\hline 1 & 2.75 & 0.75 \\
\hline 2 & 1.83 & 0.83 \\
\hline 2 & 1.83 & 0.58 \\
\hline 5 & 1.08 & 0.29 \\
\hline
\end{tabular}

Table 2: MOS for 'advertisement'

\begin{tabular}{|c|c|c|}
\hline Zapping Time(sec) & MOS & Std. Dev. \\
\hline 0 & 4.58 & 1.16 \\
\hline 0.1 & 3.00 & 1.41 \\
\hline 0.2 & 3.33 & 1.07 \\
\hline 0.2 & 3.08 & 1.24 \\
\hline 0.5 & 3.33 & 1.15 \\
\hline 0.5 & 3.16 & 0.94 \\
\hline 1 & 3.25 & 0.87 \\
\hline 2 & 2.50 & 1.09 \\
\hline
\end{tabular}

\begin{tabular}{|l|l|l|}
\hline 2 & 2.67 & 0.89 \\
\hline 5 & 1.75 & 0.87 \\
\hline
\end{tabular}

As seen from the tables, the standard deviation is lower for the MOS of the black screen experiment. This implies the opinion of the users for the black screen zapping is quite stable. However, for an advertisement related MOS the opinion of different people shows more variance.

The MOS results, together with their $95 \%$ confidence intervals, are also shown in Figure 2 and Figure 3.

QoE of Zapping (95\% Confidence Interval)

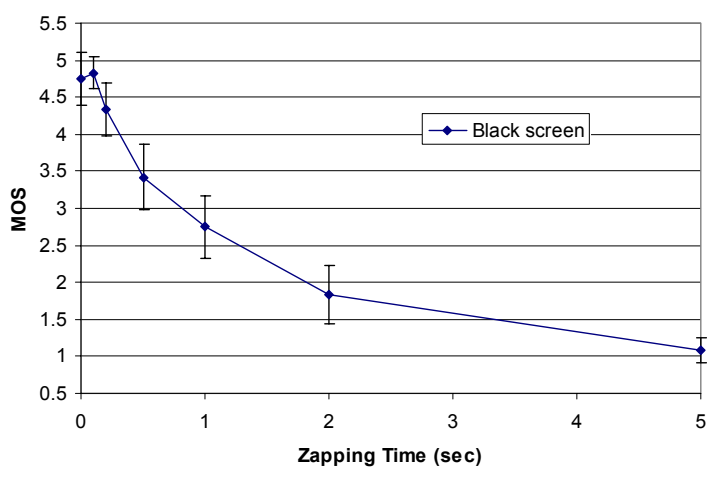

Figure 2: MOS for 'black screen'

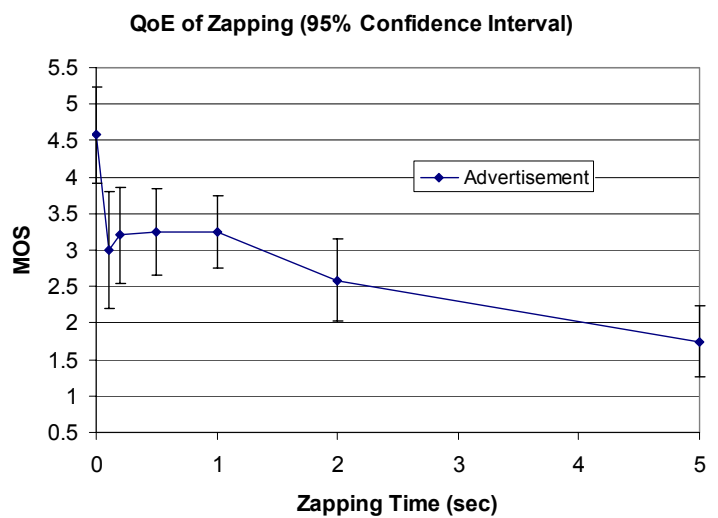

Figure 3: MOS for 'advertisement'

In order to compare the two cases, Figure 4 contains the MOS results for both 'black screen' and 'advertisement'. The following important insights can be obtained from Figure 4:

- The QoE decreases as the zapping time increases, both for 'black screen' and 'advertisement', except for 'advertisement' for zapping times between $0.1 \mathrm{sec}$ and $1 \mathrm{sec}$. 
- The MOS for 'advertisement' exceeds the MOS for 'black screen' for zapping times greater than 0.65 sec. This implies that the users prefer 'advertisement' only when the zapping time is sufficiently large. For zapping times of 1, 2 and 5 $\mathrm{sec}$, the anticipated QoE increment is clearly seen, as the 'advertisement' curve for these zapping times shifts upwards with respect to the 'black screen' curve.

- The 'advertisement' MOS is more or less constant, for zapping times less than $1 \mathrm{sec}$. However, it decreases when the zapping time increases to $2 \mathrm{sec}$ and $5 \mathrm{sec}$. This means users are still annoyed with longer zapping times, even though advertisements are shown during zapping.

- The QoE curve for 'advertisement' drops with high slope from zero zapping time to a zapping time of 0.1 sec. Because the 'black screen' curve decreases smoothly, we conclude that it is a bad idea to show advertisements in case of short zapping times.

QoE of Zapping

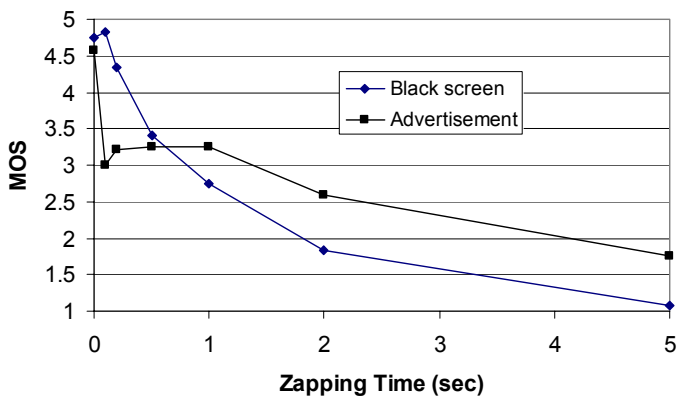

Figure 4: MOS for 'black screen' and 'advertisement'

\subsection{Comparison for 'black screen' with previous results}

The 'black screen' experiment was conducted before, see [7]. Our test scenarios are similar to the one reported in [7], except for some minor changes in the setup, like the laptop used for the experiment, the experiment room and the test subjects. The results obtained from the two tests are compared in the table below.

Table 3: 'Black screen' MOS for our experiment and previous experiment in [7]

\begin{tabular}{|c|c|c|}
\hline Zapping & MOS & MOS \\
Time(sec) & Our & Experiment \\
& experiment & in [7] \\
\hline
\end{tabular}

\begin{tabular}{|c|c|c|}
\hline 0 & 4.75 & 4.90 \\
\hline 0.1 & 4.83 & 4.90 \\
\hline 0.2 & 4.42 & 4.60 \\
\hline 0.2 & 4.25 & 4.50 \\
\hline 0.5 & 3.33 & 3.50 \\
\hline 0.5 & 3.50 & 3.30 \\
\hline 1 & 2.75 & 2.30 \\
\hline 2 & 1.83 & 1.60 \\
\hline 2 & 1.83 & 2.00 \\
\hline 5 & 1.08 & 1.10 \\
\hline
\end{tabular}

It is clear that the outcome of the experiments is almost similar. In fact, the correlation between the two experiments is as high as 0.989 .

The authors of [7] suggested the following model for the relation between zapping time (in sec) and QoE (expressed in MOS), for the 'black screen' case :

$M O S=\max \{\min \{-1.02 * \ln ($ Zapping Time $)+2.65,5\}, 1\}$.

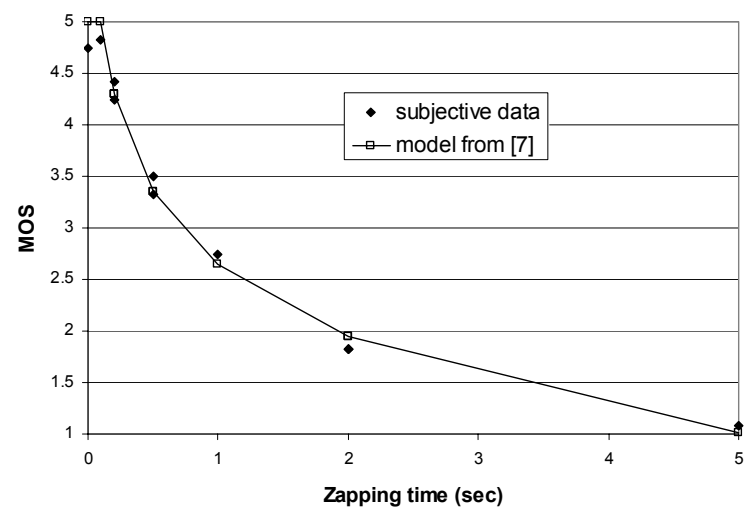

Figure 5: Comparing our 'black screen' results with the model from [7]

It is clear from Figure 5 that our 'black screen' results are in line with the model from [7].

\subsection{QoE model for 'advertisement'}

Analogous to the QoE model for 'black screen' in [7] we will now suggest a QoE model for 'advertisement'. Using curve fitting on the following intervals (in seconds) for the zapping time: [0,0.1], $[0.1,1]$ and $[1,5]$, we arrive at the following QoE model:

$$
\operatorname{MOS}=\max \left\{y_{1}, \min \left\{y_{2}, y_{3}\right\}\right\}
$$


where

$$
\begin{aligned}
& \left.y_{1}=-15.8 * \text { (Zapping Time }\right)+4.58, \\
& y_{2}=0.10 * \ln (\text { Zapping Time })+3.27 \\
& \left.y_{3}=-0.93 * \ln \text { (Zapping Time }\right)+3.27
\end{aligned}
$$

Using Table 2 we can validate the QoE model suggested in Eq. (1). This validation is visualised in Figure 6.

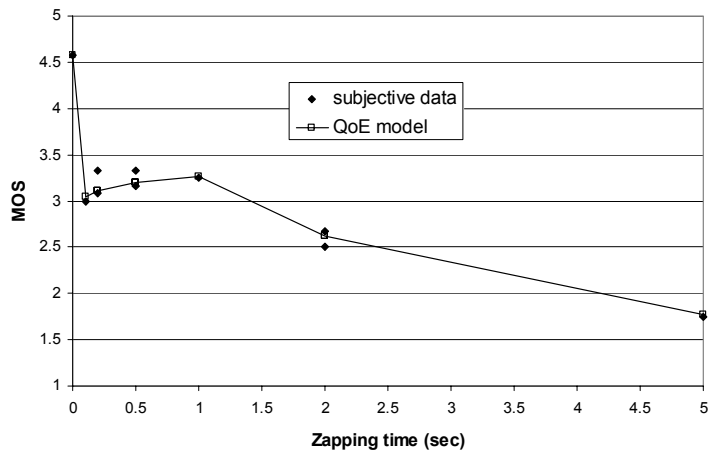

Figure 6 - MOS versus Zapping Time

It turns out that the correlation between the subjective data and the QoE model is 0.99 which is very high. In addition, the RMSE (Root Mean Square Error) equals 0.297 while the MCI (Mean Confidence Interval) satisfies 0.357 . Therefore we conclude that the QoE model given by Eq. (1) is very useful for assessing the QoE of zapping for 'advertisement'.

\subsection{Discussion on user comments}

In addition to evaluating the MOS, users were asked to comment on the usability of advertisements during the zapping times and the reasons behind the MOS scores they gave.

The following are the main comments of the users,

a) A logo advertisement is not good enough for longer zapping times: Most users get annoyed with a single picture advertisement that is displayed for 2 or 5 seconds. It is better to put a video advertisement for such long zapping delays.

b) Advertisements which have darker (non-bright) colors are better for the user perception: A white background picture advertisement is not good if the channels have a black background. So, it is good to avoid dynamic changes in the frame color.

\section{Conclusions}

Measuring the QoE of IPTV is an important issue for vendors and service providers. Channel zapping time is a major factor that affects QoE in IPTV. One of the ways to increase the user perceived quality of channel zapping, is to display advertisements during zapping, instead of the usual black screens. From our conducted subjective experiment, it is found that, for some ranges of zapping times, advertisements lead to a better QoE than black screens. However, for small zapping times black screen is found to be better. For intermediate zapping times picture advertisements are convenient. For longer zapping times picture advertisements give a better QoE than black screens; however, using video advertisements might give even better QoE in that situation. In the future we would like to conduct subjective tests where advertisement clips are used during long zapping times. This work might lead to the establishment of two zapping time thresholds: a black screen should be used below the lower threshold and video advertisements above the higher threshold. We also plan to conduct subjective tests in other countries, to see whether or not regional differences occur.

\section{Acknowledgement}

This work is partially supported by the CELTIC Project RUBENS. For more information, see http://wiki-rubens.celtic-initiative.org.

\section{References}

[1] Bigband Networks, "Methods and apparatus for using delay time during switching events to display previously stored information elements", US Patents 7237251, March 2000.

[2] N. Degrande, K. Laevens, D. De Vleeschauwer, R. Sharpe, "Increasing the user perceived quality for IPTV services", IEEE Communications Magazine, Vol. 46, Issue 2, Feb. 2008, pp. 94-100.

[3] DSL Forum, "Triple Play Services Quality of Experience (QoE) Requirements and Mechanisms", Technical Report TR-126, December 2006.

[4] Nick Fielibert, "Quality Issues in IP Delivery: Set-top boxes", September 2008,

http://www.ieee.org/organizations/society/bt/08iptv3.pdf

[5] ITU Focus group on IPTV, "Quality of Experience requirements for IPTV", FG IPTV-DOC-0118, July 2007.

[6] ITU-T Rec. P.800, "Methods for Subjective, Determination of Transmission Quality",

[7] Robert Kooij, Kamal Ahmed, Kjell Brunnström, "Perceived Quality of Channel Zapping", Proc. of the fifth IASTED International Conference, Comm. Systems and Networks, Aug. 28-30, 2006, Palma de Mallorca, Spain, pp. 155-158.

[8] Spirent communications white paper, "Delivering optimal QOE for IPTV success", Feb.2006. 